\title{
On the Geometry of Pseudo-Slant Submanifolds of a Cosymplectic Manifold
}

\author{
Süileyman Dirik* and Mehmet Atçeken \\ (Communicated by H. Hilmi HACISALİHOĞLU )
}

\begin{abstract}
In this paper, we study pseudo-slant submanifolds of a Cosymplectic manifold. We research integrability conditions for the distributions which are involved in the definition of a pseudo-slant submanifold. The necessary and sufficient conditions are given for a pseudo-slant submanifold to be pseudo-slant product.
\end{abstract}

Keywords: Cosymplectic manifold; Slant submanifold; Pseudo-slant submanifold; Pseudo-slant product.

AMS Subject Classification (2010): 53C15; 53C25; 53C42; 53D15.

\section{Introduction}

The differential geometry of slant submanifolds has shown an increasing development since B.Y. Chen defined slant submanifolds in complex manifolds as a natural generallization of both invariant and antiinvariant submanifolds $[9,10]$. After then many research articles have been appeared on the existence of these submanifolds in various know spaces. The slant submanifols of an almost contact metric manifolds were defined and studied by A. Lotta [15]. After, such submanifolds were studied in [5] and by J. L. Cabrerizo et al, of Sasakian manifolds [6].

Semi-slant submanifolds of Kaehler manifold N. Papaghich [16], as a naturel generalization of slant submanifolds. After then, bi-slant submanifolds was introduced in a almost Hermitian manifold. Recently, Carriazo defined and studied bi-slant submanifolds in an almost Hermitian manifold and gave the notion of pseudo-slant submanifold in an almost Hermitian manifold. After then, V. A. Khan and M. A. Khan [12], defined and studied the contact version of pseudo-slant submanifold in a Sasakian manifold. Recently, M. Atçeken [2] studied slant and pseudo-slant submanifold in $(L C S)_{n}$-manifolds.

The present paper is organized as follows.

In this paper, we study the geometry of the pseudo-slant submanifolds of a Cosymplectic manifold. In section 2, we review basic formulas and definitions for a Cosymplectic manifold and their submanifolds. In section 3, we recall the definition and some basic results of a pseudo-slant submanifold of almost contact metric manifold. We deal with the integrability of the distributions on the pseudo-slant submanifolds of a Cosymplectic manifold and then we obtain analogous results for these submanifolds in the setting of Cosymplectic manifolds. The necessary and sufficient conditions are given for a pseudo-slant submanifold to be pseudo-slant product.

\section{Preliminaries}

In this section, we give some notations used throughout this paper. We recall some necessary fact and formulas from the theory of Cosymplectic manifolds and their submanifols. 
Let $\widetilde{M}$ be a $(2 m+1)$-dimensional $C^{\infty}$ - differentiable manifold with the almost contact metric structure $(\phi, \xi, \eta, g)$, where $\phi$ is a tensor field of type $(1,1), \xi$ is a vector field, $\eta 1$-form and $g$ Riemannian metric on $\widetilde{M}$, satisfying

$$
\begin{gathered}
\phi^{2} X=-X+\eta(X) \xi, \\
\phi \xi=0, \quad \eta \circ \phi=0, \quad \eta(\xi)=1, \quad \eta(X)=g(X, \xi)
\end{gathered}
$$

and

$$
g(\phi X, \phi Y)=g(X, Y)-\eta(X) \eta(Y), g(\phi X, Y)=-g(X, \phi Y)
$$

for any vector fields $X, Y$ on $\widetilde{M}$.

An almost contact structure $(\phi, \xi, \eta)$ is said to be normal if the almost complex structure $J$ on the product manifold $\widetilde{M} \times R$ given by.

$$
J\left(X, f \frac{d}{d t}\right)=\left(\phi X-f \xi, \eta(X) \frac{d}{d t}\right)
$$

where $f$ is the $C^{\infty}$ - function on $\widetilde{M} \times \mathbb{R}$. The condition for normality in terms of $\phi, \xi$ and $\eta$ is $[\phi, \phi]+2 d \eta \otimes \xi=0$ on $\widetilde{M}$, where $[\phi, \phi](X, Y)=\phi^{2}[X, Y]+[\phi X, \phi Y]-\phi[\phi X, Y]-\phi[X, \phi Y]$ is the Nijenhuis tensor of $\phi$. Finally the fundamental 2 -form $\Phi$ is defined by $\Phi(X, Y)=g(X, \phi Y)$.

An almost contact metric structure $(\phi, \xi, \eta, g)$ is said to be Cosymplectic, if it is normal and both $\Phi$ and are $\eta$ closed, and structure equation of Cosymplectic manifold is given by

$$
\left(\widetilde{\nabla}_{X} \phi\right) Y=0
$$

for any vector fields $X, Y$ on $\widetilde{M}$.

Then, $\widetilde{M}$ is called a Cosymplectic manifold, where $\widetilde{\nabla}$ is the Levi-Civita connection of $g$. We have also on a Cosymplectic manifold $\widetilde{M}$

$$
\widetilde{\nabla}_{X} \xi=0
$$

for any $X, Y \in \Gamma(T \widetilde{M})$.

Now, let $M$ be a submanifold of a contact metric manifold $\widetilde{M}$ with the induced metric $g$. Also, let $\nabla$ and $\nabla^{\perp}$ be the induced connections on the tangent bundle $T M$ and the normal bundle $T^{\perp} M$ of $M$, respectively. Then the Gauss and Weingarten formulas are, respectively, given by

$$
\widetilde{\nabla}_{X} Y=\nabla_{X} Y+h(X, Y)
$$

and

$$
\widetilde{\nabla}_{X} V=-A_{V} X+\nabla_{X}^{\perp} V
$$

where $h$ and $A_{V}$ are the second fundamental form and the shape operator (corresponding to the normal vector field $V$ ), respectively, for the immersion of $M$ into $\widetilde{M}$. The second fundamental form and shape operator are related by formula

$$
g\left(A_{V} X, Y\right)=g(h(X, Y), V)
$$

for all $X, Y \in \Gamma(T M)$ and $V \in \Gamma\left(T^{\perp} M\right)$.

If $h(X, Y)=0$, for each $X, Y \in \Gamma(T M)$ then $M$ is said to be totally geodesic submanifold. 


\section{Pseudo-Slant Submanifolds of a Cosymplectic Manifold}

In this section, we will obtain the integrability condition of the distributions of pseudo-slant submanifold of a Cosymplectic manifold. Also, the necessary and sufficient conditions are given for a pseudo-slant submanifold to be pseudo-slant product.

Now, let $M$ be a submanifold of an almost contact metric manifold $\widetilde{M}$. Then for any $X \in \Gamma(T M)$, we can write

$$
\phi X=T X+N X,
$$

where $T X$ is the tangential component and $N X$ is the normal component of $\phi X$. Similarly, for $V \in \Gamma\left(T^{\perp} M\right)$, we can write

$$
\phi V=t V+n V,
$$

where $t V$ is the tangential component and $n V$ is also the normal component of $\phi V$.

Thus by using (2.1), (3.1) and (3.2), we obtain

$$
T^{2}=-I+\eta \otimes \xi-t N, \quad N T+n N=0
$$

and

$$
T t+t n=0, \quad N t+n^{2}=-I .
$$

Furthermore, for any $X, Y \in \Gamma(T M)$, we have $g(T X, Y)=-g(X, T Y)$ and $V, U \in \Gamma\left(T^{\perp} M\right)$, we get $g(U, n V)=$ $-g(n U, V)$. These show that $T$ and $n$ are also skew-symmetric tensor fields. Moreover, for any $X \in \Gamma(T M)$ and $V \in \Gamma\left(T^{\perp} M\right)$, we have

$$
g(N X, V)=-g(X, t V),
$$

which gives the relation between $N$ and $t$.

Furthermore, the covariant derivatives of the tensor field $T, N, t$ and $n$ are, respectively, defined by

$$
\begin{gathered}
\left(\nabla_{X} T\right) Y=\nabla_{X} T Y-T \nabla_{X} Y, \\
\left(\nabla_{X} N\right) Y=\nabla_{X}^{\perp} N Y-N \nabla_{X} Y, \\
\left(\nabla_{X} t\right) V=\nabla_{X} t V-t \nabla_{X}^{\perp} V
\end{gathered}
$$

and

$$
\left(\nabla_{X} n\right) V=\nabla_{X}^{\perp} n V-n \nabla_{X}^{\perp} V
$$

A submanifold $M$ is said to be invariant if $N$ is identically zero, that is, $\phi X \in \Gamma(T M)$ for all $X \in \Gamma(T M)$. On the other hand, $M$ is said to be anti- invariant if $T$ is identically zero, that is, $\phi X \in \Gamma\left(T^{\perp} M\right)$ for all $X \in \Gamma(T M)$. By an easy computation, we obtain the following formulas

$$
\left(\nabla_{X} T\right) Y=A_{N Y} X+\operatorname{th}(X, Y)
$$

and

$$
\left(\nabla_{X} N\right) Y=n h(X, Y)-h(X, T Y) .
$$

Similarly, for any $V \in \Gamma\left(T^{\perp} M\right)$ and $X \in \Gamma(T M)$, we obtain

$$
\left(\nabla_{X} t\right) V=A_{n V} X-T A_{V} X
$$


and

$$
\left(\nabla_{X} n\right) V=-h(t V, X)-N A_{V} X .
$$

Since $M$ is tangent to $\xi$, making use of (2.5), (2.6), (2.8) and (3.1), we obtain

$$
\nabla_{X} \xi=0, h(X, \xi)=0, A_{V} \xi=0
$$

for all $V \in \Gamma\left(T^{\perp} M\right)$ and $X \in \Gamma(T M)$.

In contact geometry, A. Lotta introduced slant submanifold as follows [15].

Definition 3.1. A submanifold $M$ of an almost contact metric manifold $\widetilde{M}$ is said to be a slant submanifold if for any $x \in M$ and $X \in T_{x}(M)-\xi$, the angle between $\phi X$ and $T_{x}(M)$ is constant. The constant angle $\theta(x) \in\left[0, \frac{\pi}{2}\right]$ is called slant angel of $M$ in $\widetilde{M}$. If $\theta=0$ the submanifold is invariant submanifold, if $\theta=\frac{\pi}{2}$ then it is anti-invariant submanifold, if $\theta \neq\left\{0, \frac{\pi}{2}\right\}$ then it is proper slant submanifold. [15]. The tangent bundle $T M$ of $M$ is decomposed as $T M=D \oplus \xi$, where the orthogonal complementary distribution $D$ of $\xi$ is know as the slant distribution on $M$. We have the following result in the setting of almost contact manifolds given by Cabrerizo et.al.

Theorem 3.1. Let $M$ be a slant submanifold of an almost contact metric manifold $\widetilde{M}$ such that $\xi \in \Gamma(T M)$. Then, $M$ is slant submanifold if and only if there exists a constant $\lambda \in[0,1]$ such that

$$
T^{2}=-\lambda(I-\eta \otimes \xi)
$$

furthermore, in this case, if $\theta$ is the slant angle of $M$, then $\lambda=\cos ^{2} \theta[6]$.

Corollary 3.1. Let $M$ be a slant submanifold of an almost contact metric manifold $\widetilde{M}$ with slant angle $\theta$. Then for any $X, Y \in \Gamma(T M)$, we have

$$
g(T X, T Y)=\cos ^{2} \theta\{g(X, Y)-\eta(X) \eta(Y)\}
$$

and

$$
g(N X, N Y)=\sin ^{2} \theta\{g(X, Y)-\eta(X) \eta(Y)\} .
$$

It is well known that $t h=0$ plays an important role in the geometry of submanifolds. This means that the induced structure $T$ is a cosymplectic structure on $M$. By using (3.10) and (3.14), we obtain

$$
\eta\left(\left(\nabla_{X} T\right) Y\right)=0
$$

for $X, Y \in \Gamma\left(D_{\theta}\right)$.

Definition 3.2. Let $M$ be a submanifold of an almost contact metric manifold $\widetilde{M} . M$ is said to be pseudo-slant of $\widetilde{M}$ if there exist two orthogonal distributions $D^{\perp}$ and $D_{\theta}$ on $M$ such that:

i) TM has the orthogonal direct decomposition $T M=D^{\perp} \oplus D_{\theta}, \xi \in \Gamma\left(D_{\theta}\right)$.

ii) The distribution $D^{\perp}$ is an anti-invariant, that is, $\phi D^{\perp} \subset T^{\perp} M$.

iii) The distribution $D_{\theta}$ is a slant, that is, the slant angle between of $D_{\theta}$ and $\phi\left(D_{\theta}\right)$ is a constant.

If $\theta=0$ then, the submanifold becomes a semi-invariant submanifold.

Let $m_{1}=\operatorname{dim}\left(D^{\perp}\right)$ and $m_{2}=\operatorname{dim}\left(D_{\theta}\right)$. We distinguish the following five cases.

i) If $m_{2}=0$ or $\theta=\frac{\pi}{2}$, then $M$ is an anti-invariant submanifold.

ii) If $m_{1}=0$ and $\theta=0$, then $M$ is invariant submanifold.

iii) If $m_{1}=0$ and $\theta \neq 0, \frac{\pi}{2}$, then $M$ is a proper slant submanifold.

iv) If $m_{2} m_{1} \neq 0$ and $\theta=0$, then $M$ is a semi-invariant submanifold.

v) If $m_{2} m_{1} \neq 0$ and $\theta \neq 0, \frac{\pi}{2}$, then $M$ is a pseudo-slant submanifold [12].

If we denote the projections on $D^{\perp}$ and $D_{\theta}$ by $P_{1}$ and $P_{2}$, respectively, then for any vector field $X \in \Gamma(T M)$, we can write

$$
X=P_{1} X+P_{2} X+\eta(X) \xi
$$


Now operating $\phi$ on both sides of equation (3.19), we have

$$
\phi X=\phi P_{1} X+\phi P_{2} X
$$

and

$$
T X+N X=N P_{1} X+T P_{2} X+N P_{2} X
$$

We can easily to see

$$
T X=T P_{2} X, \quad N X=N P_{1} X+N P_{2} X
$$

and

$$
\phi P_{1} X=N P_{1} X, T P_{1} X=0, \phi P_{2} X=T P_{2} X+N P_{2} X, T P_{2} X \in \Gamma\left(D_{\theta}\right) .
$$

If we denote the orthogonal complementary of $\phi(T M)$ in $T^{\perp} M$ by $\mu$, then the normal bundle $T^{\perp} M$ can be decomposed as follows

$$
T^{\perp} M=N\left(D^{\perp}\right) \oplus N\left(D_{\theta}\right) \oplus \mu .
$$

We can easily see that the bundle $\mu$ is an invariant subbundle with respect to $\phi$. Since $D^{\perp}$ and $D_{\theta}$ are orthogonal distribution on $M, g(Z, X)=0$ for each $Z \in \Gamma\left(D^{\perp}\right)$ and $X \in \Gamma\left(D_{\theta}\right)$. Thus, by equation (2.3) and (3.1), we can write

$$
g(N Z, N X)=g(\phi Z, \phi X)=g(Z, X)=0
$$

that is, the distributions $N\left(D^{\perp}\right)$ and $N\left(D_{\theta}\right)$ are also mutually perpendicular. In fact, the decomposition (3.20) is an orthogonal direct decomposition.

Theorem 3.2. Let $M$ be a submanifold of an almost contact metric manifold $\widetilde{M}$. Then $D_{\theta}$ is slant distribution if only and if there is a constant $\lambda \in[0,1]$ such that

$$
\left(T P_{2}\right)^{2} X=-\lambda X
$$

for any $X \in \Gamma\left(D_{\theta}\right)$. In this case, the slant angle $\theta$ satisfies $\lambda=\cos ^{2} \theta[6]$.

Now, we construct on example of a pseudo-slant submanifold in an almost contact metric manifold.

Example 3.1. Let $M$ be a submanifold of $\mathbb{R}^{7}$ defined by the equation

$$
(u, v, s, t, z)=(\sqrt{3} u, v, v \sin \alpha, v \cos \alpha, s \cos t,-s \cos t, z) .
$$

We can easily to see that the tangent bundle of $M$ is spanned by the tangent vectors

$$
\begin{aligned}
& e_{1}=\sqrt{3} \frac{\partial}{\partial x_{1}}, \quad e_{2}=\frac{\partial}{\partial y_{1}}+\sin \alpha \frac{\partial}{\partial x_{2}}+\cos \alpha \frac{\partial}{\partial y_{2}} \\
& e_{3}=\cos t \frac{\partial}{\partial x_{3}}-\cos t \frac{\partial}{\partial y_{3}}, e_{4}=-s \sin t \frac{\partial}{\partial x_{3}}+s \sin t \frac{\partial}{\partial y_{3}} \\
& e_{5}=\xi=\frac{\partial}{\partial z} .
\end{aligned}
$$

For the contact structure $\phi$ of $\mathbb{R}^{7}$, choosing

$$
\begin{aligned}
\phi\left(\frac{\partial}{\partial x_{i}}\right) & =\frac{\partial}{\partial y_{i}}, \quad \phi\left(\frac{\partial}{\partial y_{j}}\right)=-\frac{\partial}{\partial x_{j}}, \quad 1 \leq i, j \leq 3 \\
\phi\left(\frac{\partial}{\partial z}\right) & =0, \xi=\frac{\partial}{\partial z}, \quad \eta=d z .
\end{aligned}
$$

For any vector field $W=\mu_{i} \frac{\partial}{\partial x_{i}}+\nu_{j} \frac{\partial}{\partial y_{j}}+\lambda \frac{\partial}{\partial z} \in T\left(\mathbb{R}^{7}\right)$, then we have

$$
\phi W=\mu_{i} \phi\left(\frac{\partial}{\partial x_{i}}\right)+\nu_{j} \phi\left(\frac{\partial}{\partial y_{j}}\right)+\lambda \phi\left(\frac{\partial}{\partial z}\right)=\mu_{i} \frac{\partial}{\partial y_{j}}-\nu_{j} \frac{\partial}{\partial x_{i}},
$$




$$
\begin{gathered}
g(\phi W, \phi W)=g\left(\mu_{i} \frac{\partial}{\partial y_{j}}-\nu_{j} \frac{\partial}{\partial x_{i}}, \mu_{i} \frac{\partial}{\partial y_{j}}-\nu_{j} \frac{\partial}{\partial x_{i}}\right)=\mu_{i}^{2}+\nu_{j}^{2}, \\
g(W, W)=g\left(\mu_{i} \frac{\partial}{\partial x_{i}}+\nu_{j} \frac{\partial}{\partial y_{j}}+\lambda \frac{\partial}{\partial z}, \mu_{i} \frac{\partial}{\partial x_{i}}+\nu_{j} \frac{\partial}{\partial y_{j}}+\lambda \frac{\partial}{\partial z}\right)=\mu_{i}^{2}+\nu_{j}^{2}+\lambda^{2}, \\
\eta(W)=g(W, \xi)=g\left(\mu_{i} \frac{\partial}{\partial x_{i}}+\nu_{j} \frac{\partial}{\partial y_{j}}+\lambda \frac{\partial}{\partial z}, \frac{\partial}{\partial z}\right)=\lambda
\end{gathered}
$$

and

$$
\phi^{2} W=-\mu_{i} \frac{\partial}{\partial x_{i}}-\nu_{j} \frac{\partial}{\partial y_{j}}-\lambda \frac{\partial}{\partial z}+\lambda \frac{\partial}{\partial z}=-W+\eta(W) \xi
$$

for any $i, j=1,2,3$. It follows that $g(\phi W, \phi W)=g(W, W)-\eta^{2}(W)$. Thus $(\phi, \xi, \eta, g)$ is an almost contact metric structure on $\mathbb{R}^{7}$. We call the usual contact metric structure of $\mathbb{R}^{7}$. Then we have

$$
\begin{aligned}
\phi e_{1} & =\sqrt{3} \frac{\partial}{\partial y_{1}}, \phi e_{2}=-\frac{\partial}{\partial x_{1}}+\sin \alpha \frac{\partial}{\partial y_{2}}-\cos \alpha \frac{\partial}{\partial x_{2}} \\
\phi e_{3} & =\cos t \frac{\partial}{\partial y_{3}}+\cos t \frac{\partial}{\partial x_{3}}, \phi e_{4}=-s \sin t \frac{\partial}{\partial y_{3}}-s \sin t \frac{\partial}{\partial x_{3}} .
\end{aligned}
$$

By direct calculations, we can infer $D_{\theta}=\operatorname{span}\left\{e_{1}, e_{2}\right\}$ is a slant distribution with slant angle $\cos \theta=\frac{g\left(e_{2}, \phi e_{1}\right)}{\left\|e_{2}\right\|\left\|\phi e_{1}\right\|}=\frac{\sqrt{2}}{2}, \theta=45^{\circ}$. Since

$$
\begin{aligned}
& g\left(\phi e_{3}, e_{1}\right)=g\left(\phi e_{3}, e_{2}\right)=g\left(\phi e_{3}, e_{4}\right)=g\left(\phi e_{3}, e_{5}\right)=0, \\
& g\left(\phi e_{4}, e_{1}\right)=g\left(\phi e_{4}, e_{2}\right)=g\left(\phi e_{4}, e_{3}\right)=g\left(\phi e_{4}, e_{5}\right)=0,
\end{aligned}
$$

$\phi e_{3}$ and $\phi e_{4}$ are orthogonal to $M$ and $D^{\perp}=\operatorname{span}\left\{e_{3}, e_{4}\right\}$ is an anti-invariant distribution. Thus $M$ is a 5 dimensional proper pseudo-slant submanifold of $\mathbb{R}^{7}$ with it's usual almost contact metric structure.

Moreover, for any $Z, W \in \Gamma\left(D^{\perp}\right)$ and $U \in \Gamma(T M)$, also by using (2.4), (2.7) and (2.8), we have

$$
\begin{aligned}
g\left(A_{N Z} W-A_{N W} Z, U\right)= & g(h(W, U), N Z)-g(h(Z, U), N W) \\
= & g\left(\widetilde{\nabla}_{U} W, \phi Z\right)-g\left(\widetilde{\nabla}_{U} Z, \phi W\right) \\
= & g\left(\phi \widetilde{\nabla}_{U} Z, W\right)-g\left(\phi \widetilde{\nabla}_{U} W, Z\right) \\
= & g\left(\widetilde{\nabla}_{U} \phi Z-\left(\widetilde{\nabla}_{U} \phi\right) Z, W\right) \\
& +g\left(\left(\widetilde{\nabla}_{U} \phi\right) W-\widetilde{\nabla}_{U} \phi W, Z\right) \\
= & g\left(\widetilde{\nabla}_{U} \phi Z, W\right)-g\left(\widetilde{\nabla}_{U} \phi W, Z\right) \\
= & -g\left(A_{N Z} U, W\right)+g\left(A_{N W} U, Z\right) \\
= & g\left(A_{N W} Z-A_{N Z} W, U\right) .
\end{aligned}
$$

It follows that

$$
A_{N Z} W=A_{N W} Z
$$

Theorem 3.3. Let $M$ be pseudo-slant submanifold of Cosymplectic manifold $\widetilde{M}$, then

for any $Z, W \in \Gamma\left(D^{\perp}\right)$.

$$
\nabla_{W}^{\perp} N Z-\nabla_{Z}^{\perp} N W \in N\left(D^{\perp}\right)
$$

Proof. For any $Z, W \in \Gamma\left(D^{\perp}\right)$ and $V \in \mu$, we have

$$
\begin{aligned}
g\left(\nabla_{W}^{\perp} N Z-\nabla_{Z}^{\perp} N W, V\right)= & g\left(\widetilde{\nabla}_{W} \phi Z+A_{\phi Z} W-\widetilde{\nabla}_{Z} \phi W-A_{\phi W} Z, V\right) \\
= & g\left(\widetilde{\nabla}_{W} \phi Z-\widetilde{\nabla}_{Z} \phi W, V\right) \\
= & g\left(\left(\widetilde{\nabla}_{W} \phi\right) Z+\phi \widetilde{\nabla}_{W} Z, V\right) \\
& -g\left(\left(\widetilde{\nabla}_{Z} \phi\right) W+\phi \widetilde{\nabla}_{Z} W, V\right) \\
= & g\left(\phi \widetilde{\nabla}_{W} Z, V\right)-g\left(\phi \widetilde{\nabla}_{Z} W, V\right) \\
= & g\left(\widetilde{\nabla}_{Z} W, \phi V\right)-g\left(\widetilde{\nabla}_{W} Z, \phi V\right) \\
= & g\left(\nabla_{Z} W, \phi V\right)-g\left(\nabla_{W} Z, \phi V\right) \\
& +g(h(Z, W), \phi V)-g(h(W, Z), \phi V)=0 .
\end{aligned}
$$


Thus the proof is complete.

Theorem 3.4. Let $M$ be a pseudo-slant submanifold of a Cosymplectic manifold $\widetilde{M}$. Then the anti-invariant distribution $D^{\perp}$ is completely integrable and its maximal integral submanifold is an anti-invariant submanifold of $\widetilde{M}$.

Proof. For any $Z, W \in \Gamma\left(D^{\perp}\right)$ and $X \in \Gamma\left(D_{\theta}\right)$, by using (2.4), (2.6), (2.7) and (2.8), we have

$$
\begin{aligned}
g([Z, W], X)= & g\left(\widetilde{\nabla}_{Z} W-\widetilde{\nabla}_{W} Z, X\right)=g\left(\widetilde{\nabla}_{W} X, Z\right)-g\left(\widetilde{\nabla}_{Z} X, W\right) \\
= & g\left(\phi \widetilde{\nabla}_{W} X, \phi Z\right)-g\left(\phi \widetilde{\nabla}_{Z} X, \phi W\right) \\
= & g\left(\widetilde{\nabla}_{W} \phi X, \phi Z\right)-g\left(\widetilde{\nabla}_{Z} \phi X, \phi W\right) \\
& -g\left(\left(\widetilde{\nabla}_{W} \phi\right) X, \phi Z\right)+g\left(\left(\widetilde{\nabla}_{Z} \phi\right) X, \phi W\right) \\
= & g\left(\widetilde{\nabla}_{W} T X+\widetilde{\nabla}_{W} N X, N Z\right) \\
& -g\left(\widetilde{\nabla}_{Z} T X+\widetilde{\nabla}_{Z} N X, N W\right) \\
= & g(h(T X, W), N Z)-g(h(T X, Z), N W) \\
& +g\left(\nabla_{W}^{\perp} N X, N Z\right)-g\left(\nabla_{Z}^{\perp} N X, N W\right) \\
= & g\left(A_{N Z} W-A_{N W} Z, T X\right)+g\left(\nabla_{W}^{\perp} N X, N Z\right) \\
& -g\left(\nabla_{Z}^{\perp} N X, N W\right)
\end{aligned}
$$

by using (3.7), (3.11) and (3.22), we have

$$
\begin{aligned}
g([Z, W], X)= & g\left(\nabla_{W}^{\perp} N X, N Z\right)-g\left(\nabla_{Z}^{\perp} N X, N W\right) \\
= & g\left(\left(\nabla_{W} N\right) X+N \nabla_{W} X, N Z\right) \\
& -g\left(\left(\nabla_{Z} N\right) X+N \nabla_{Z} X, N W\right) \\
= & g(n h(W, X)-h(W, T X), N Z) \\
& -g(n h(Z, X)-h(Z, T X), N W) \\
& +g\left(N \nabla_{W} X, N Z\right)-g\left(N \nabla_{Z} X, N W\right) \\
= & -g(h(W, T X), N Z)+g(h(Z, T X), N W) \\
& +g\left(N \nabla_{W} X, N Z\right)-g\left(N \nabla_{Z} X, N W\right)
\end{aligned}
$$

by using (3.17), we obtain

$$
\begin{aligned}
g([Z, W], X) & =\sin ^{2} \theta g\left(\nabla_{W} X, Z\right)-\sin ^{2} \theta g\left(\nabla_{Z} X, W\right) \\
& =\sin ^{2} \theta g\left(\nabla_{Z} W, X\right)-\sin ^{2} \theta g\left(\nabla_{W} Z, X\right) \\
& =\sin ^{2} \theta g([Z, W], X)
\end{aligned}
$$

hence

$$
\cos ^{2} \theta g([Z, W], X)=0 .
$$

Thus $[Z, W] \in \Gamma\left(D^{\perp}\right)$ for any $Z, W \in \Gamma\left(D^{\perp}\right)$, that is, anti-invariant distribution $D^{\perp}$ is always integrable and its integral submanifold is an anti-invariant submanifold of $\widetilde{M}$. Thus the proof is complete.

Now, by using (2.4), we have

$$
\left(\widetilde{\nabla}_{X} \phi\right) Y=\widetilde{\nabla}_{X} \phi Y-\phi \widetilde{\nabla}_{X} Y=0 .
$$

Hence, by using (2.6), (2.7), (3.1) and (3.2), we obtain

$$
-A_{N Y} X+\nabla_{X}^{\perp} N Y-T \nabla_{X} Y-N \nabla_{X} Y-\operatorname{th}(X, Y)-n h(X, Y)=0 .
$$

for any $X, Y \in \Gamma\left(D^{\perp}\right)$. From the tangent components of this last equation, we obtain

$$
A_{N Y} X+T \nabla_{X} Y+\operatorname{th}(X, Y)=0 .
$$

By interchange roles of $X$ and $Y$ in (3.23), we have

$$
A_{N X} Y+T \nabla_{Y} X+\operatorname{th}(X, Y)=0
$$


which is equivalent to

$$
T[X, Y]=A_{N X} Y-A_{N Y} X .
$$

From (3.22), we can easily to see that the anti-invariant distribution $D^{\perp}$ is always integrable.

Since the ambient manifold $\widetilde{M}$ is Cosymplectic, for any $Z, W \in \Gamma\left(D^{\perp}\right)$

$$
\left(\widetilde{\nabla}_{Z} \phi\right) W=0
$$

which implies that

$$
\widetilde{\nabla}_{Z} \phi W-\phi \widetilde{\nabla}_{Z} W=\widetilde{\nabla}_{Z} N W-\phi\left(\nabla_{Z} W+h(W, Z)\right)=0
$$

So we have

$$
-A_{N W} Z+\nabla \frac{1}{Z} N W-T \nabla_{Z} W-N \nabla_{Z} W-\operatorname{th}(W, Z)-n h(W, Z)=0 .
$$

From the tangent components of the last equation, we obtain

$$
A_{N W} Z+T \nabla_{Z} W+\operatorname{th}(W, Z)=0 .
$$

From the above equation, we conclude

$$
T[W, Z]=A_{N W} Z+T \nabla_{W} Z+\operatorname{th}(W, Z) .
$$

The anti-invariant distribution $D^{\perp}$ is in integrable, $\phi[Z, W]=N[Z, W]$ because of the tangent component of $\phi[Z, W]$ is zero. So we have

$$
A_{N W} Z+T \nabla_{W} Z+\operatorname{th}(W, Z)=0 .
$$

Similarly, we obtain

$$
A_{N Z} W+T \nabla_{Z} W+\operatorname{th}(Z, W)=0 .
$$

Here, by using (3.22), (3.25) and (3.26), we obtain

$$
\left(\nabla_{Z} T\right) W=\left(\nabla_{W} T\right) Z
$$

Lemma 3.1. Let $M$ be a pseudo-slant submanifold of a Cosymplectic manifold $\widetilde{M}$. Then we have

$$
\left(\nabla_{Z} T\right) W=\left(\nabla_{W} T\right) Z
$$

for any $Z, W \in \Gamma\left(D^{\perp}\right)$.

Theorem 3.5. Let $M$ be a pseudo-slant submanifold of a Cosymplectic manifold $\widetilde{M}$. Then the slant distribution $D_{\theta}$ is integrable if and only if

$$
P_{1}\left\{\nabla_{X} T Y-T \nabla_{Y} X-A_{N Y} X-t h(X, Y)\right\}=0
$$

for any $X, Y \in \Gamma\left(D_{\theta}\right)$.

Proof. For any $X, Y \in \Gamma\left(D_{\theta}\right)$, by using (2.4) and considering the tangential component, we obtain

$$
T[X, Y]=\nabla_{X} T Y-T \nabla_{Y} X-A_{N Y} X-\operatorname{th}(X, Y) .
$$

Applying $P_{1}$ to (3.29), we get (3.28)

Theorem 3.6. Let $M$ be a pseudo-slant submanifold of a Cosymplectic manifold $\widetilde{M}$. Then the slant distribution $D_{\theta}$ is integrable if and only if

$$
\nabla_{Z}^{\perp} N W-\nabla_{W}^{\perp} N Z+h(Z, T W)-h(W, T Z) \in \mu \oplus N\left(D_{\theta}\right)
$$

for any $Z, W \in \Gamma\left(D_{\theta}\right)$.

Proof. For any $Z, W \in \Gamma\left(D_{\theta}\right)$ and $X \in \Gamma\left(D^{\perp}\right)$, by using (2.3), we have

$$
\begin{aligned}
g([Z, W], X) & =g\left(\widetilde{\nabla}_{Z} W, X\right)-g\left(\widetilde{\nabla}_{W} Z, X\right) \\
& =g\left(\phi \widetilde{\nabla}_{Z} W, \phi X\right)+\eta\left(\widetilde{\nabla}_{Z} W\right) \eta(X) \\
& -g\left(\phi \widetilde{\nabla}_{W} Z, \phi X\right)-\eta\left(\widetilde{\nabla}_{W} Z\right) \eta(X) .
\end{aligned}
$$


Thus we obtain

$$
\begin{aligned}
g([Z, W], X)= & \left.g\left(\widetilde{\nabla}_{Z} \phi W, N X\right)-g\left(\widetilde{\nabla}_{Z} \phi\right) W, N X\right) \\
& \left.-g\left(\widetilde{\nabla}_{W} \phi Z, N X\right)+\left(\widetilde{\nabla}_{W} \phi\right) Z, N X\right) .
\end{aligned}
$$

Taking into account (2.4) and (3.1), we have

$$
g([Z, W], X)=g\left(\widetilde{\nabla}_{Z}(T W+N W), N X\right)-g\left(\widetilde{\nabla}_{W}(T Z+N Z), N X\right) .
$$

Then from the Gauss and Weingarten formulas the above equation takes the form, we have

$$
\begin{aligned}
g([Z, W], X) & =g(h(Z, T W), N X)+g\left(\nabla_{Z}^{\perp} N W, N X\right) \\
& -g(h(W, T Z), N X)-g\left(\nabla_{W}^{\perp} N Z, N X\right) .
\end{aligned}
$$

Since, we have $N X \in N\left(D^{\perp}\right) \subseteq T^{\perp} M$ we conclude

$$
\nabla_{Z}^{\perp} N W-\nabla_{W}^{\perp} N Z+h(Z, T W)-h(W, T Z) \in \mu \oplus N\left(D_{\theta}\right) .
$$

Theorem 3.7. Let $M$ be a pseudo-slant submanifold of a Cosymplectic manifold $\widetilde{M}$. Then the slant distribution $D_{\theta}$ is integrable if and only if

$$
T A_{N U} X+A_{N U} T X=0
$$

for any $U \in \Gamma\left(D^{\perp}\right)$ and $X \in \Gamma\left(D_{\theta}\right)$.

Proof. For any $U \in \Gamma\left(D^{\perp}\right)$ and $X, Y \in \Gamma\left(D_{\theta}\right)$, by direct calculation, we have

$$
\begin{aligned}
g([X, Y], U) & =g\left(\widetilde{\nabla}_{X} Y-\widetilde{\nabla}_{Y} X, U\right) \\
& =g\left(\phi \widetilde{\nabla}_{X} Y, \phi U\right)-g\left(\phi \widetilde{\nabla}_{Y} X, \phi U\right) \\
& =g\left(\phi \widetilde{\nabla}_{X} Y, N U\right)-g\left(\phi \widetilde{\nabla}_{Y} X, N U\right) \\
& =g\left(\widetilde{\nabla}_{X} \phi Y, N U\right)-g\left(\widetilde{\nabla}_{Y} \phi X, N U\right) \\
& -g\left(\left(\widetilde{\nabla}_{X} \phi\right) Y, N U\right)+g\left(\left(\widetilde{\nabla}_{Y} \phi\right) X, N U\right) .
\end{aligned}
$$

Hence, by using (2.4) and (3.1), we obtain

$$
\begin{aligned}
g([X, Y], U) & =g\left(\widetilde{\nabla}_{Y} N U, \phi X\right)-g\left(\widetilde{\nabla}_{X} N U, \phi Y\right) \\
& =g\left(\widetilde{\nabla}_{Y} N U, T X\right)+g\left(\widetilde{\nabla}_{Y} N U, N X\right) \\
& -g\left(\widetilde{\nabla}_{X} N U, T Y\right)-g\left(\widetilde{\nabla}_{X} N U, N Y\right) .
\end{aligned}
$$

On the other hand, from (2.4), (2.6) and (2.7), we have

$$
\begin{aligned}
\left(\widetilde{\nabla}_{X} \phi\right) U & =\widetilde{\nabla}_{X} \phi U-\phi \widetilde{\nabla}_{X} U \\
0 & =\widetilde{\nabla}_{X} N U-T \nabla_{X} U-N \nabla_{X} U-\operatorname{th}(X, U)-n h(X, U)
\end{aligned}
$$

that is,

$$
-A_{N U} X+\nabla_{X}^{\perp} N U=T \nabla_{X} U+N \nabla_{X} U+\operatorname{th}(X, U)+n h(X, U) .
$$

From the tangential components, we obtain

$$
-A_{N U} X=T \nabla_{X} U+\operatorname{th}(X, U)
$$

and

$$
\left(\nabla_{X} N\right) U=n h(X, U) .
$$


Also, by using (3.7) and (3.30) we conclude that

$$
\begin{aligned}
g([X, Y], U) & =g\left(A_{N U} X, T Y\right)-g\left(A_{N U} Y, T X\right)+g\left(\nabla_{Y}^{\perp} N U, N X\right)-g\left(\nabla_{X}^{\perp} N U, N Y\right) \\
& =-g\left(T A_{N U} X, Y\right)-g\left(A_{N U} T X, Y\right)+g\left(\left(\nabla_{Y} N\right) U+N \nabla_{Y} U, N X\right) \\
& -g\left(\left(\nabla_{X} N\right) U+N \nabla_{X} U, N Y\right) \\
& =-g\left(T A_{N U} X, Y\right)-g\left(A_{N U} T X, Y\right)+g(n h(Y, U), N X)+g\left(N \nabla_{Y} U, N X\right) \\
& -g(n h(X, U), N Y)-g\left(N \nabla_{X} U, N Y\right) \\
& =-g\left(T A_{N U} X, Y\right)-g\left(A_{N U} T X, Y\right)+g\left(N \nabla_{Y} U, N X\right)-g\left(N \nabla_{X} U, N Y\right) \\
& =-g\left(T A_{N U} X, Y\right)-g\left(A_{N U} T X, Y\right)+\sin ^{2} \theta\left\{g\left(\nabla_{Y} U, X\right)-g\left(\nabla_{X} U, Y\right)\right\} \\
& =-g\left(T A_{N U} X, Y\right)-g\left(A_{N U} T X, Y\right)+\sin ^{2} \theta\left\{g\left(\nabla_{X} Y, U\right)-g\left(\nabla_{Y} X, U\right)\right\} \\
& =-g\left(T A_{N U} X, Y\right)-g\left(A_{N U} T X, Y\right)+\sin ^{2} \theta\{g([X, Y], U)\} .
\end{aligned}
$$

So we conclude

$$
\cos ^{2} \theta\{[X, Y], U\}=-g\left(T A_{N U} X, Y\right)-g\left(A_{N U} T X, Y\right)
$$

which verifies our assertion.

For a pseudo-slant submanifold $M$ of $\widetilde{M}$, the slant and anti- invariant distributions are totally geodesic in $M$, then $M$ is called pseudo-slant product.

The following theorem characterize the pseudo-slant product in Cosymplectic manifolds.

Theorem 3.8. Let $M$ be a pseudo-slant submanifold of a Cosymplectic manifold $\widetilde{M}$. Then $M$ is a pseudo-slant product if and only if the second fundamental form $h$ satisfies

$$
\operatorname{th}(X, Z)=0
$$

for all $X \in \Gamma\left(D_{\theta}\right)$ and $Z \in \Gamma(T M)$.

Proof. For all $X, Y \in \Gamma\left(D_{\theta}\right)$ and $U, V \in \Gamma\left(D^{\perp}\right)$, we have

$$
\begin{aligned}
g\left(\nabla_{X} Y, U\right) & =-g\left(\nabla_{X} U, Y\right)=-g\left(\widetilde{\nabla}_{X} U, Y\right) \\
& =-g\left(\phi \widetilde{\nabla}_{X} U, \phi Y\right)-\eta\left(\widetilde{\nabla}_{X} U\right) \eta(Y) \\
& =g\left(\left(\widetilde{\nabla}_{X} \phi\right) U-\widetilde{\nabla}_{X} \phi U, \phi Y\right) \\
& -g\left(\nabla_{X} U+h(X, U), \xi\right) \eta(Y) \\
& =-g\left(\widetilde{\nabla}_{X} \phi U, \phi Y\right)-g\left(\nabla_{X} U, \xi\right) \eta(Y) \\
& =-g\left(\widetilde{\nabla}_{X} \phi U, \phi Y\right)+g\left(\nabla_{X} \xi, U\right) \eta(Y) \\
& =-g\left(\widetilde{\nabla}_{X} \phi U, T Y\right)-g\left(\widetilde{\nabla}_{X} \phi U, N Y\right) .
\end{aligned}
$$

$\phi U=N U$ and using (3.14), we obtain

$$
g\left(\nabla_{X} Y, U\right)=-g\left(\widetilde{\nabla}_{X} N U, T Y\right)-g\left(\widetilde{\nabla}_{X} N U, N Y\right) .
$$

Using (2.6) and (2.7), we have

$$
\begin{aligned}
g\left(\nabla_{X} Y, U\right) & =g\left(A_{N U} X-\nabla_{X}^{\perp} N U, T Y\right)+g\left(A_{N U} X-\nabla_{X}^{\perp} N U, N Y\right) \\
& =g\left(A_{N U} X, T Y\right)-g\left(\left(\nabla_{X} N\right) U, N Y\right)-g\left(N \nabla_{X} U, N Y\right) \\
& =g\left(A_{N U} X, T Y\right)-g\left(N \nabla_{X} U, N Y\right)-g(n h(X, U), N Y)
\end{aligned}
$$

hence using (3.14) and (3.17), we have

$$
\begin{aligned}
g\left(\nabla_{X} Y, U\right) & =g\left(A_{N U} X, T Y\right)-g\left(N \nabla_{X} U, N Y\right) \\
& =g\left(A_{N U} X, T Y\right)-\sin ^{2} \theta\left\{g\left(\nabla_{X} U, Y\right)-\eta\left(\nabla_{X} U\right) \eta(Y)\right\} \\
& =g(h(X, T Y), N U)-\sin ^{2} \theta g\left(\nabla_{X} U, Y\right)+\sin ^{2} \theta g\left(\nabla_{X} U, \xi\right) \eta(Y) \\
& =g(h(X, T Y), N U)+\sin ^{2} \theta g\left(\nabla_{X} Y, U\right)-\sin ^{2} \theta g\left(\nabla_{X} \xi, U\right) \eta(Y) \\
& =g(h(X, T Y), N U)+\sin ^{2} \theta g\left(\nabla_{X} Y, U\right)
\end{aligned}
$$


that is

$$
\cos ^{2} \theta g\left(\nabla_{X} Y, U\right)=g(h(X, T Y), N U)=-g(t h(X, T Y), U) .
$$

In the same way, we obtain

$$
\begin{aligned}
g\left(\nabla_{V} U, X\right) & =g\left(\widetilde{\nabla}_{V} U, X\right)=-g\left(\widetilde{\nabla}_{V} X, U\right) \\
& =-g\left(\phi \widetilde{\nabla}_{V} X, \phi U\right)-\eta\left(\widetilde{\nabla}_{V} X\right) \eta(U) \\
& =g\left(\left(\widetilde{\nabla}_{V} \phi\right) X, \phi U\right)-g\left(\widetilde{\nabla}_{V} \phi X, \phi U\right)
\end{aligned}
$$

For $U, V \in \Gamma\left(D^{\perp}\right)$, since the tangent component of $\phi U$ and $T U$ are zero, we have

$$
\begin{aligned}
g\left(\nabla_{V} U, X\right) & =-g\left(\widetilde{\nabla}_{V} \phi X, N U\right)+g\left(\left(\widetilde{\nabla}_{V} \phi\right) X, N U\right) \\
& =-g\left(\widetilde{\nabla}_{V} \phi X, N U\right)=-g\left(\widetilde{\nabla}_{V} T X, N U\right)-g\left(\widetilde{\nabla}_{V} N X, N U\right) \\
& =-g\left(\nabla_{V} T X+h(T X, V), N U\right)+g\left(A_{N X} V-\nabla_{V}^{\perp} N X, N U\right) \\
& =-g(h(T X, V), N U)-g\left(\nabla_{V}^{\perp} N X, N U\right) \\
& =-g(h(T X, V), N U)-g\left(\left(\nabla_{V} N\right) X+N \nabla_{V} X, N U\right)
\end{aligned}
$$

hence using (3.14), we have

$$
\begin{aligned}
g\left(\nabla_{V} U, X\right) & =-g(h(V, T X), N U)-g\left(N \nabla_{V} X, N U\right) \\
& +g(h(V, T X), N U)-g(n h(V, X), N U) \\
& =-g\left(N \nabla_{V} X, N U\right)-g(n h(V, X), N U) \\
& =-g(n h(V, X), N U)+\sin ^{2} \theta g\left(\nabla_{V} U, X\right)
\end{aligned}
$$

that is

$$
\cos ^{2} \theta g\left(\nabla_{V} U, X\right)=-g(n h(V, X), N U)=g(\operatorname{th}(V, X), U) .
$$

From equation(3.32) and (3.33). Thus $D_{\theta}$ and $D^{\perp}$ are totally geodesic in $M$ if and only if (3.31) is satisfied.

Theorem 3.9. Let $M$ be a pseudo-slant submanifold of a Cosymplectic manifold $\widetilde{M}$. If $N$ is parallel on $D_{\theta}$, then either $M$ is a $D_{\theta}$-geodesic submanifold or $h(X, Y)$ is an eigenvector of $n^{2}$ with eigenvalue $-\cos ^{2} \theta$, for any $X, Y \in \Gamma\left(D_{\theta}\right)$.

Proof. For any $X, Y \in \Gamma\left(D_{\theta}\right)$, from (3.11), we have

$$
n h(X, Y)-h(X, T Y)=0 .
$$

On the other hand, since $D_{\theta}$ is a slant distribution, we obtain

$$
\begin{aligned}
0 & =n h(X, Y-\eta(Y) \xi)-h(X, T(Y-\eta(Y) \xi)) \\
& =n h(X, Y-\eta(Y) \xi)-h(X, T Y)
\end{aligned}
$$

that is

$$
n h(X, Y-\eta(Y) \xi)=h(X, T Y) .
$$

Now, applying $n$ to (3.35), we have

$$
n^{2} h(X, Y-\eta(Y) \xi)=n h(X, T Y) .
$$

On the other hand, by interchanging of $Y$ and $T Y$ in (3.34), we have

$$
n h(X, T Y)=h\left(X, T^{2} Y\right) \text {. }
$$

Hence, using (3.15), we obtain

$$
n^{2} h(X, Y-\eta(Y) \xi)=n h(X, T Y)=h\left(X, T^{2} Y\right)=-\cos ^{2} \theta h(X, Y-\eta(Y) \xi) .
$$

This implies that either $h$ vanishes on $D_{\theta}$ or $h$ is an eigenvector of $n^{2}$ with eigenvalue $-\cos ^{2} \theta$. 


\section{Acknowledgments}

The authors are grateful to the referee(s) for the useful comments, which improved the presentation of the manuscript.

\section{References}

[1] Atçeken, M. and Dirik, S., On contact CR-submanifolds of Kenmotsu manifolds, Acta Universitatis Sapientiae, 4(2012), $182-198$.

[2] Atçeken M. and Hui, S. K., Slant and pseudo-slant submanifolds in $(L C S)_{n}$-manifolds, Czechoslovak Mathematical Journal, 63(2013), 177190.

[3] Atçeken, M. and Dirik, S., On the geometry of pseudo-slant submanifolds of a Kenmotsu manifold, Gulf joural of mathematics, 2(2014), 51-66.

[4] Blair, D., Contact manifolds in Riemannian geometry, Lecture Notes in Mathematic Springer-Verlog, New York, 509(1976).

[5] Carriazo, A., New developments in slant submanifolds theory, Narasa Publishing Hause, New Delhi. india, 2000.

[6] Cabrerizo, J. L. Carriazo, A. Fernandez L. M. and Fernandez, M., Slant submanifolds in Sasakian manifolds, Glasgow Mathematical Journal, 42(2000), 125-138.

[7] Cabrerizo, J. L. Carriazo, A. Fernandez, L. M. and Fernandez, M., Slant submanifolds in Sasakian manifolds, Geomeatriae Dedicata, 78(1999), 183-199.

[8] Chand De. U. and. Sarkar, A., On pseudo-slant submanifolds of trans-Sasakian manifolds, Proceedings of the Estonian Academy of Sciences, 60(2011), no.1, 1-11.

[9] Chen, B.-Y., Geometry of slant submanifolds, Katholieke Universiteit Leuven, Leuven, Belgium, View at Zentralblatt Math., 1990.

[10] Chen, B.-Y., Slant immersions, Bulletin of the Australian Mathematical Society, 41(1990), 135-147.

[11] Dirik, S. and Atçeken, M., Pseudo-slant submanifolds of a nearly Cosymplectic manifold, Turkish Journal of Mathematics Computer Science, Article ID 20140035,14 page (2014).

[12] Khan, V. A and. Khan, M. A., Pseudo-slant submanifolds of a Sasakian manifold, Indian Journal of Pure and Applied Mathematics, 38(2007), 31-42.

[13] Khan, M. A. Uddin, S. and Singh, K., classification on totally umbilical proper slant and hemi-slant submanifolds of a nearly transSasakian manifold, Differential Differential Geometry - Dynamical Systems, 13(2011), 117-127.

[14] Khan, M. A., Totally umbilical hemi slant submanifolds of Cosymplectic manifolds, Mathematica Aeterna, 3(2013), no. 8, 845-853.

[15] Lotta, A., Slant submanifolds in contact geometry, Bulletin Mathematical Society Roumanie, 39(1996), 183-198.

[16] Papaghuic, N., Semi-slant submanifolds of a Kaehlarian manifold, An. St. Univ. Al. I. Cuza. Univ. Iasi, 40(2009), 55-61.

[17] Uddin, S. Ozel, C. Khan, M. A. and Singh, K., Some classification result on totally umbilical proper slant and hemi slant submanifolds of a nearly Kenmotsu manifold, international journal of physical Scienses, 7(2012), 5538-5544.

[18] Uddin, S. Bernardine, W. R. and. Mustafa, A. A., Warped product pseudo-slant submanifolds of a nearly Cosymplectic manifold, Hindawi Publishing Corporation Abstract and Applied Analysis, Volume , Article ID 420890, 13 pp, doi:10.1155/2012/420890 (2012).

\section{Affiliations}

\section{SÜLEYMAN DIRIK}

AdDRESS: Amasya University, Dept. of Statistics, 05000, Amasya-Turkey.

E-MAIL: suleyman.dirik@amasya.edu.tr,E-MAIL: slymndirik@gmail.com

\section{MeHmet AtÇEKEN}

ADDRESS: Gaziosmanpasa University, Dept. of Mathematics, 60100, Tokat-Turkey.

E-MAIL: mehmet.atceken382@gmail.com 